\title{
Vitamin B2 lung-marking method using black light irradiation
}

\author{
Yusuke Tanaka ${ }^{1}$, Isao Matsumoto ${ }^{1}$, Munehisa Takata $^{1}$, Daisuke Saito ${ }^{1}$, Shuhei Yoshida ${ }^{1}$, Masaya Tamura $^{1}$, \\ Wataru Koda $^{2}$, Ryuichi Waseda ${ }^{3}$, Hirofumi Takemura ${ }^{1}$
}

${ }^{1}$ Department of Thoracic, Cardiovascular and General Surgery, Kanazawa University, Kanazawa, Japan; ${ }^{2}$ Department of Radiology, Kanazawa University Graduate School of Medical Sciences, Kanazawa, Japan; ${ }^{3}$ Department of Thoracic Surgery, Faculty of Medicine, Fukuoka University, Fukuoka, Japan

Contributions: (I) Conception and design: Y Tanaka, I Matsumoto; (II) Administrative support: I Matsumoto; (III) Provision of study materials or patients: Y Tanaka, M Takata; (IV) Collection and assembly of data: Y Tanaka, M Takata, D Saito, S Yoshida, W Koda; (V) Data analysis and interpretation: Y Tanaka, M Takata, D Saito, S Yoshida; (VI) Manuscript writing: All authors; (VII) Final approval of manuscript: All authors.

Correspondence to: Yusuke Tanaka, MD. Department of Thoracic, Cardiovascular and General Surgery, Kanazawa University, 13-1 Takara-machi, Kanazawa 920-8641, Japan. Email: yusuke.t.0613@gmail.com.

Background: Various approaches and markers for marking the lungs prior to lung tumor resection have been reported. In clinical practice, the hook wire localization method is often used owing to the simplicity of the technique. However, although rare, this method is associated with air embolism, which can be lethal. Because vitamin B2 is harmless to the body and fluorescent, it was applied to various methods for thoracic surgery. Using a pig model, we aimed to examine whether a lung-marking method involving the injection of vitamin B2 to peripheral small lung lesions and observing them under black light irradiation could replace the hook wire localization method.

Methods: We used a pig model to perform hook wire localization of the lungs and at the same time injected $1 \mathrm{~mL}$ of a vitamin B2 aqueous solution to the lung parenchyma at the hook wire puncture site under the visceral pleura. Subsequently, we measured the length of the fluorescent marked area and fluorescence intensity over time. Black light was used to assess the fluorescent marked area, and fluorescence intensity was quantified using image analysis software.

Results: Lung-marking was successful in all five pigs and we visualized the vitamin B2-marked area under black light irradiation. Measurements were taken immediately after thoracotomy (0 min) and 60 and $120 \mathrm{~min}$ thereafter. No changes in the length of the marked area $(1.3 \pm 0.3 / 1.2 \pm 0.3 / 1.1 \pm 0.3 \mathrm{~cm}, 0 / 60 / 120 \mathrm{~min}, \mathrm{P}=0.21)$ and fluorescence intensity $(162.8 \pm 55.1 / 157.2 \pm 63.1 / 165.2 \pm 62.2,0 / 60 / 120 \mathrm{~min}, \mathrm{P}=0.96)$ were observed over time. Compared to the non-marked area (normal lungs), the marked area showed significantly higher fluorescence intensity ( $\mathrm{P}=0.01 / 0.01 / 0.01,0 / 60 / 120 \mathrm{~min})$.

Conclusions: Vitamin B2 lung-marking was performed safely and accurately using the pig model, providing good visibility of the marked area. This approach may replace the hook wire localization method. In the near future, we plan to conduct clinical trials to evaluate the applicability of this method in humans.

Keywords: Lung-marking; vitamin B2; black light

Submitted Aug 31, 2019. Accepted for publication Dec 13, 2019.

doi: $10.21037 /$ jtd.2020.01.06

View this article at: http://dx.doi.org/10.21037/jtd.2020.01.06

\section{Introduction}

Small lung lesions without pleural changes that are difficult to palpate are subjected to lung-marking prior to lung tumor resection, to better visualize the location of the tumor. To date, various lung-marking methods are being studied. In clinical practice, the percutaneous computed tomography (CT)-guided hook wire localization method is often used, owing to the simplicity of the technique $(1,2)$. In Japan, the Guiding-Marker System (GMS, Hakko Co., Ltd., Nagano, Japan) is used for around 2,000 cases 
annually. Although rare, the hook wire localization method is associated with air embolism, which can be lethal (3). Given that vitamin B2 is harmless to the body and is fluorescent, we have reported various methods to apply it to thoracic surgery (4-6). In this study, we aimed to establish a facile and safe lung-marking method and proposed a novel lung-marking method of injecting a vitamin B2 aqueous solution under the visceral pleura. We assessed this method with the idea of applying vitamin B2 marking to human clinical practices in the future, and to that effect, we aimed to evaluate whether this method can replace the existing hook wire localization method, through animal experiments using adult pigs.

\section{Methods}

\section{Preparation of animals}

General anesthesia was induced in five adult pigs (Sus scrofa domestica, aged 2.5 months and weighed approximately 30 kg; Sankyo Labo Service Co., Inc., Tokyo, Japan) by intramuscular injection of ketamine hydrochloride (20 mg/kg, Ketalar, Sankyo Co., Ltd., Tokyo, Japan). General anesthesia was maintained by inhalation of sevoflurane (0.5-2\%, Maruishi Pharmaceutical Co., Ltd., Osaka, Japan) or intravenous injection of pancuronium bromide $(0.1 \mathrm{mg} / \mathrm{kg}$, Musculax, Schering-Plough K.K., Osaka, Japan). Tracheostomy was performed with the animal in a supine position, and the airway was secured using an endobronchial tube (24 Fr, Fuji Systems Co., Tokyo, Japan) before performing the marking by switching the animal to a left lateral decubitus position.

\section{Fluorescence substance}

Vitamin B2 is a non-allergic, non-toxic substance that is classified as a water-soluble vitamin. In this study, we used $0.01 \%$ flavin adenine dinucleotide (FAD) sodium (Fravitan, Toa Eiyo Ltd., Fukushima, Japan) as the vitamin B2 aqueous solution. It has been used as a supplement for patients with vitamin B2 deficiency and a prophylactic agent for migraine headache. In recent years, it has been evaluated as a potential adjuvant therapy for cancer and certain eye disorders $(7,8)$.

\section{Photodynamic diagnosis}

We used a black light to evaluate the fluorescence of the vitamin B2-marked site. Black light is also referred to as UV-A light, Wood's light, or ultraviolet light. Using Europium-doped strontium borohydride $\left(\mathrm{SrB}_{4} \mathrm{O}_{7} \mathrm{~F}: \mathrm{Eu}^{2+}\right)$ or lead-doped barium silicide $\left(\mathrm{BaSi}_{2} \mathrm{O}_{5}: \mathrm{Pb}^{+}\right)$as a fluorescent substance, the black light irradiated invisible light (wavelength: $315-400 \mathrm{~nm}$ ) with the peak wavelength mainly at $365 \mathrm{~nm}$, cutting visible light of $400 \mathrm{~nm}$ or higher using Wood's glass. In this study, we assessed the fluorescence of vitamin B2 using an electric stand-type black light irradiator with a peak wavelength of $365 \mathrm{~nm}$ (Sankyo Denki, Co., Ltd., Tokyo, Japan).

\section{Marking procedure}

Although lung dissection in adult pigs has been reported, in this study, we marked the lungs under X-ray fluoroscopicguidance in the dorsal region of the pig, which corresponds to the lower lobe of the lungs in humans (9). The chest wall of adult pigs is very thick; thus, a sufficient amount of time is required when marking the lungs from the external body surface, to enable observation of the thoracic cavity. For this reason, we made an incision in the skin and peeled it, from the subcutaneous tissue to the muscle layer, before performing the marking procedure with the ribs and intercostal muscles exposed. To evaluate whether vitamin B2 marking is as effective as hook wire localization, we performed two types of marking sequentially on the same site. For hook wire localization, we used the GMS, which is used in the clinical context for preoperative marking of lung tumors. The GMS is made from a guiding needle (size $18 \mathrm{G}, 30 \mathrm{~mm}$ long) to penetrate the chest wall and a marking needle (size $21 \mathrm{G}, 100 \mathrm{~mm}$ long) to puncture the visceral pleura. The marking needle contains a stainless hook wire $(0.28 \mathrm{~mm}$ wide, $10 \mathrm{~mm}$ long) connected to a nylon thread and a pusher that is used to push the hook wire. We connected a guide sheath equipped with a hemostatic valve (Catheter Introducer, Medikit Co., Ltd., Tokyo, Japan) to the guiding needle and filled the inner cavity of the guiding needle with FAD sodium beforehand to ensure that air does not enter during puncturing. Using the guiding needle, we first punctured the visceral pleura under $\mathrm{X}$-ray fluoroscopy (Figure 1A,B). Then, under X-ray fluoroscopicguidance, using the marking needle, we punctured from the guide sheath equipped with a hemostatic valve, which was connected to the guiding needle (Figure 2A,B). By pushing the pusher inside the marking needle, the hook wire was placed $1 \mathrm{~cm}$ under the visceral pleura, and then by lightly tugging on the nylon thread connected to the hook wire, 


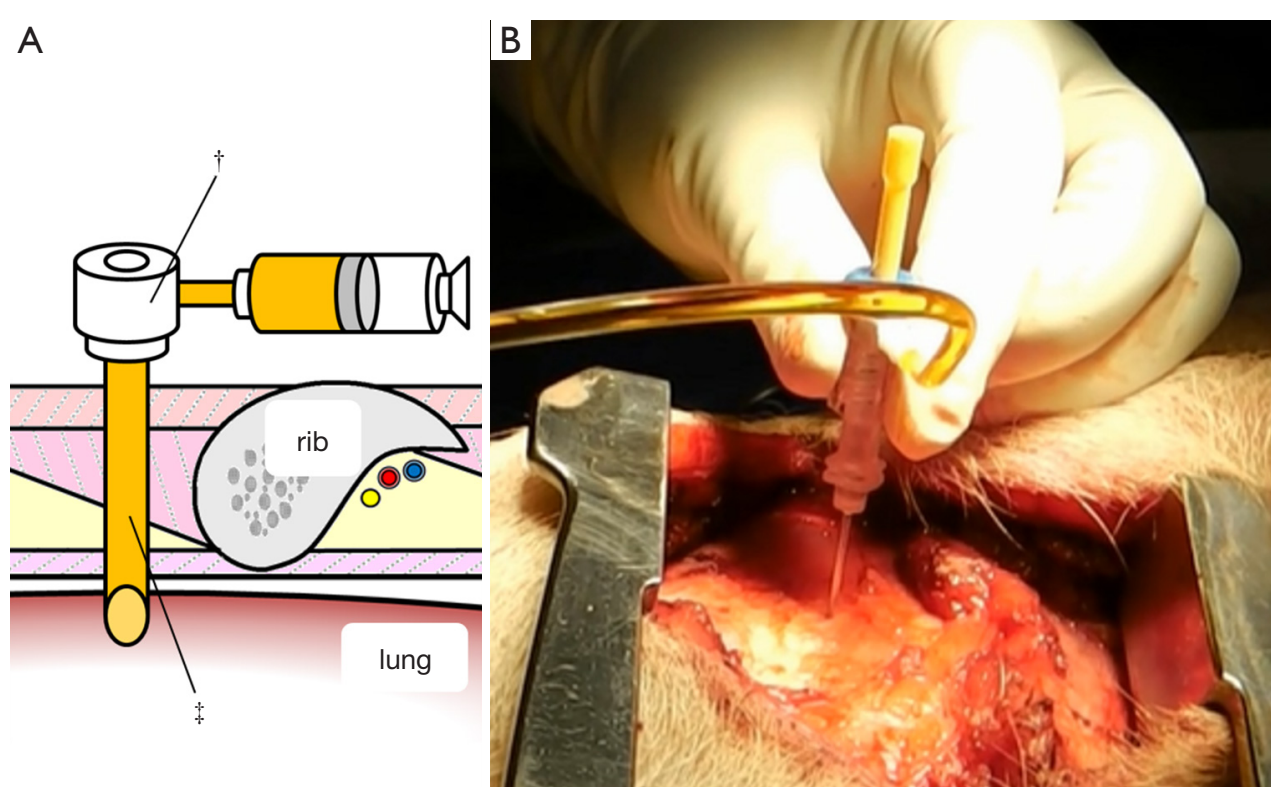

Figure 1 Connect the guide sheath equipped with a hemostatic valve $(\dagger)$ to the guiding needle $(\dagger)$ and puncture the lung parenchyma with the inner cavity of the needle filled with the vitamin B2 solution under X-ray fluoroscopic-guidance (A: schema; B: experiments using the pig model).

we confirmed that the hook wire had been securely placed in the lung. After removing the marking needle, $1 \mathrm{~mL}$ of FAD sodium was injected into the hook wire puncture site using the guiding needle (Figure $3 A, B$ ). After the injection, the intercostal spaces at the puncture site were opened to observe the marking area.

\section{Fluorescence evaluation}

We checked the regions marked with vitamin B2 using the nylon thread connected to the hook wire as a marker and then evaluated the length and fluorescence intensity at the marking area by irradiating the same site with a black light. The evaluation took place immediately after thoracotomy $(0 \mathrm{~min})$ and 60 and $120 \mathrm{~min}$ thereafter. We directly measured the marked area to determine its length. Fluorescence intensity was determined by first recording the surgical field irradiated with black light using a digital video camera (HX-WA 20-H, Panasonic Corporation, Osaka, Japan) and then analyzing the obtained footage/image using image analysis software (U11437, Hamamatsu photonics Co., Ltd., Hamamatsu, Japan). This analysis software is capable of digitizing the luminance of the marked and non-marked areas. Luminance is determined based on the weighted average of the RGB value and is expressed as an integer from 0 to 255 with a combination of red, green, and blue values (black being 0 , white being 255). We compared the luminance of the marked area to that of the non-marked area after digitizing them.

\section{Safety evaluation}

Immediately after lung-marking, we checked the animals' vital signs, oxygen saturation, and the presence of air leakage and bleeding from the lungs at the puncture site.

\section{Animal care}

The experimental animals were handled in accordance with the "Guidelines for the Care and Use of Laboratory Animals" (approval no. 153644) stipulated by the Kanazawa University, as well as the "Guide for the Care and Use of Laboratory Animals" (Version 8) announced by the National Research Council in 2011. All experimental animals were euthanized using an intravenous administration of potassium chloride under general anesthesia, after the experiment.

\section{Statistical analysis}

All results are presented as mean and standard deviation, and comparative examinations were performed using MannWhitney $U$ tests. One-way repeated measured analysis of 
A

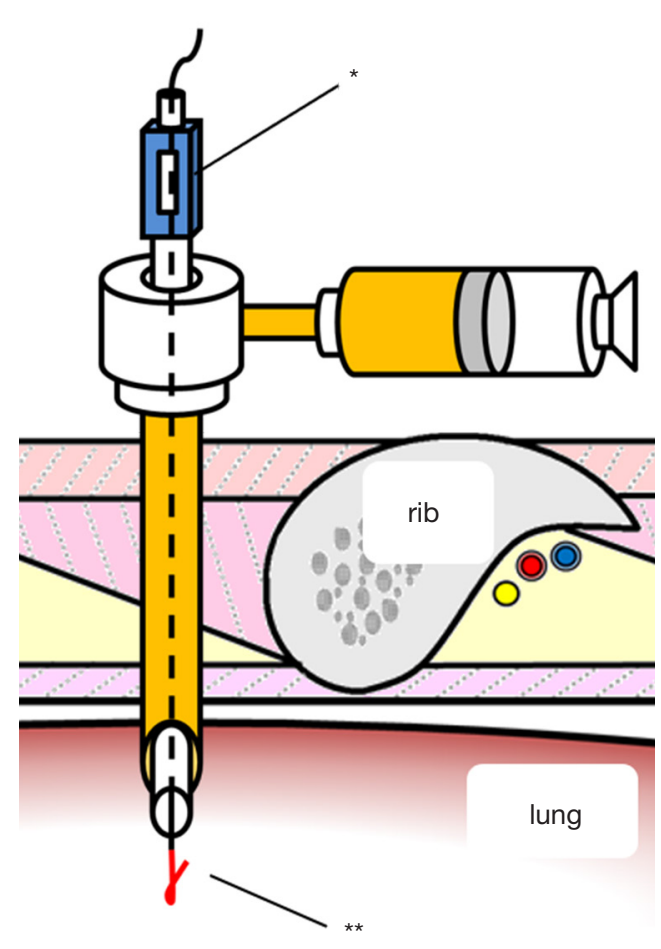

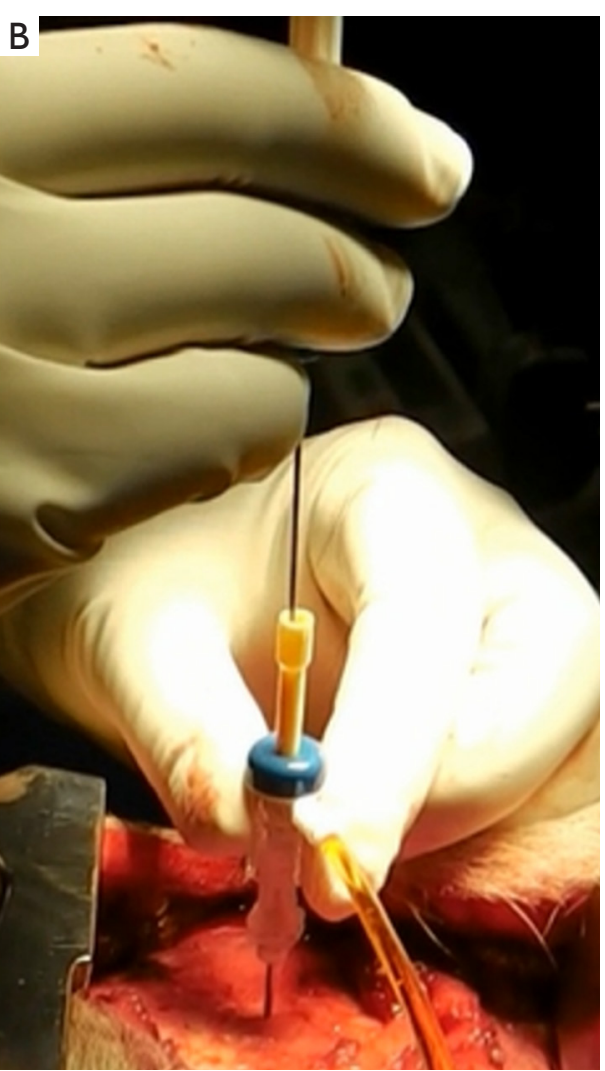

Figure 2 Using the marking needle $\left(^{*}\right)$, puncture the lung parenchyma and place the hook wire $\left.{ }^{* *}\right)($ A: schema; B: experiments using the pig model).
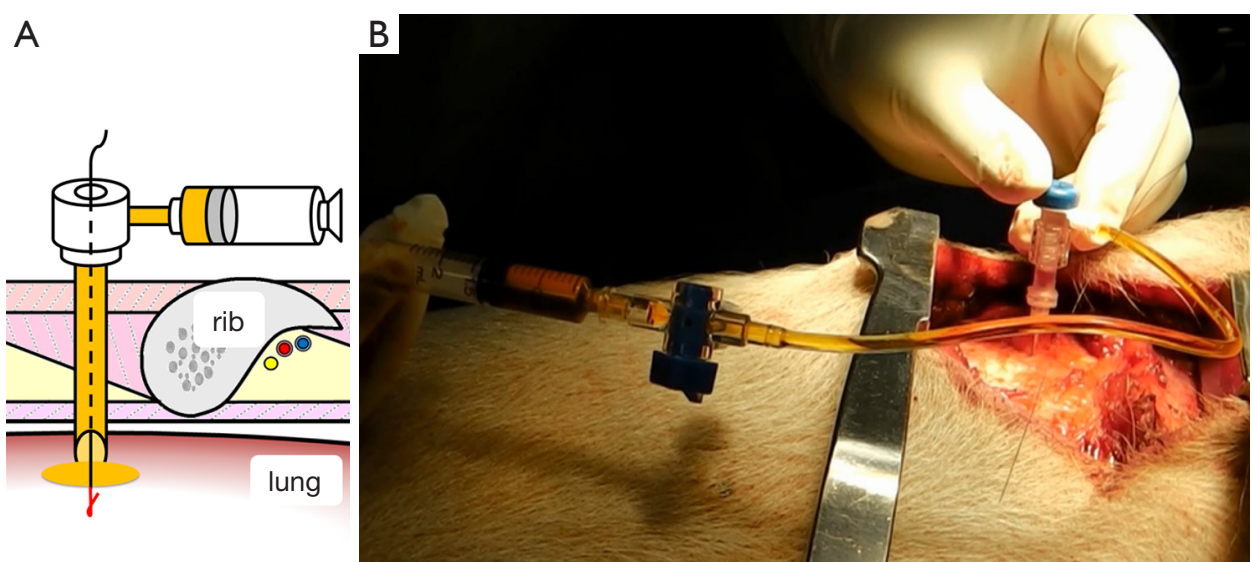

Figure 3 After removing the marking needle, inject the vitamin B2 solution around the hook wire (A: schema; B: experiments using the pig model).

variance was used for comparison of continuous variables. STATMATE (ATMS Co., Ltd., Tokyo, Japan) was used for data analysis, with $\mathrm{P}<0.05$ considered to indicate a statistically significant difference.

\section{Results}

All five animals underwent lung-marking as planned. The hook wire was placed inside the lungs and the lungs were checked for vitamin B2 leakage. There was neither air 

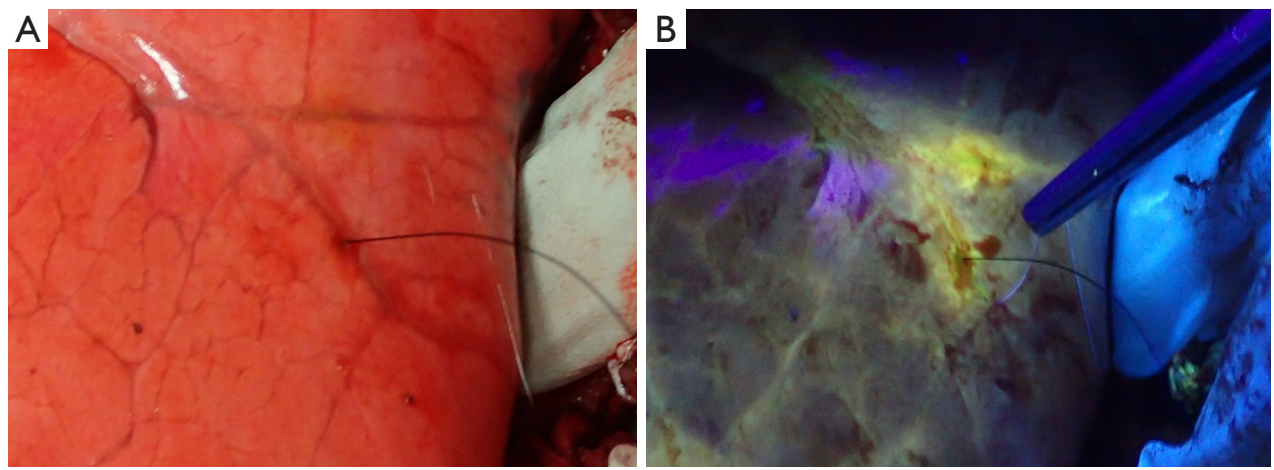

Figure 4 After hook wire localization and vitamin B2 marking. (A) Normal light imaging; (B) fluorescence imaging.

leakage nor bleeding from the puncture site in the lungs or changes in vital signs until the end of the observation. Thoracotomy was performed on an average of $12 \pm 3 \mathrm{~min}$ after vitamin B2 marking until the observation of the marked area. In all five adult pigs, we could identify the marked area around the hook wire and observe fluorescence using the black light (Figure 4). The length of the marked area at 0,60 , and $120 \mathrm{~min}$ after thoracotomy was $1.3 \pm 0.3$, $1.2 \pm 0.3$, and $1.1 \pm 0.3 \mathrm{~cm}$, respectively, and the fluorescence did not attenuate for more than $2 \mathrm{~h}$ after the marking $(\mathrm{P}=0.21$, Figure 5). The fluorescence intensity of the marked area at 0,60 , and $120 \mathrm{~min}$ after thoracotomy was $162.8 \pm 55.1,157.2 \pm 63.1$, and $165.2 \pm 62.2$, respectively. The fluorescence intensity in the non-marked area was $34.4 \pm 26.1,22.6 \pm 21.6$, and $30.6 \pm 22.1$, respectively. All observed values in the marked area were significantly greater than those of the non-marked area $(\mathrm{P}=0.01 / 0.01 / 0.01$, $0 / 60 / 120 \mathrm{~min}$, respectively). Furthermore, the intensity of the marked and non-marked areas did not change between the first measurement immediately after thoracotomy to $120 \mathrm{~min}$ after thoracotomy $(\mathrm{P}=0.96 / 0.18$, marking/nonmarking, Figure 6). The fluorescence in the marked area was maintained for a while even after $120 \mathrm{~min}$.

\section{Discussion}

In this study, we used a pig model to evaluate the efficacy of a novel lung-marking method that uses vitamin B2, a fluorescent substance that is non-toxic in humans. This approach to lung-marking allows more than $2 \mathrm{~h}$ of localized and accurate visualization of the location of a target lesion, with reduced risk of complications related to lung puncture and reduced risk of air embolism in particular.

In recent years, the use of high-resolution CT has led to the discovery of many small lung lesions, such as ground- glass opacities, which do not show pleural changes and are difficult to palpate during surgery. The purpose of lung-marking is to visualize the location of tumors that cannot be palpated during surgery. In addition, because of the increasing difficulty of palpation accompanied by the advancement of minimally invasive surgery and the finding that reductive surgery can provide good prognosis for early-stage lung cancer patients with low lung function $(10,11)$, there is a growing need for an accurate method of preoperative lung-marking. Gaining correct understanding of the position of the lesion during surgery is not only helpful for conducting lung tumor resection with sufficient tumor margin but also for shortening the operating time, avoiding conversion to thoracotomy, and preserving respiratory function.

To date, various reports have focused on the marker and marking approaches for preoperative lung-marking. Hook wire $(1,2)$, dyes such as indigo carmine (12-14) and ICG (15), as well as contrast agents like barium and lipiodol (16), and a metallic coil $(17,18)$ have been used as markers, while percutaneous or transbronchial methods have been selected as marking approaches.

For percutaneous approaches, a hook wire, dyes, contrast agents, and so on, are used as markers, and the merits include simple and accurate marking $(1,2,13,16)$, and implementation is possible with existing CT devices. Contrarily, the association of bleeding and pneumothorax as complications of lung puncture, and the presence of anatomically inaccessible parts, such as the pulmonary apex, the mediastinum, interlobar spaces, backside of the shoulder blade, and diaphragm surface, are some of the known problems of this approach (1-3). When a hook wire is used as a marker, there is a risk of dropping or losing it (1-3). Although rare, air embolism, with an incidence rate of $0.24 \%$ (1), can cause a stroke or myocardial infarction, 


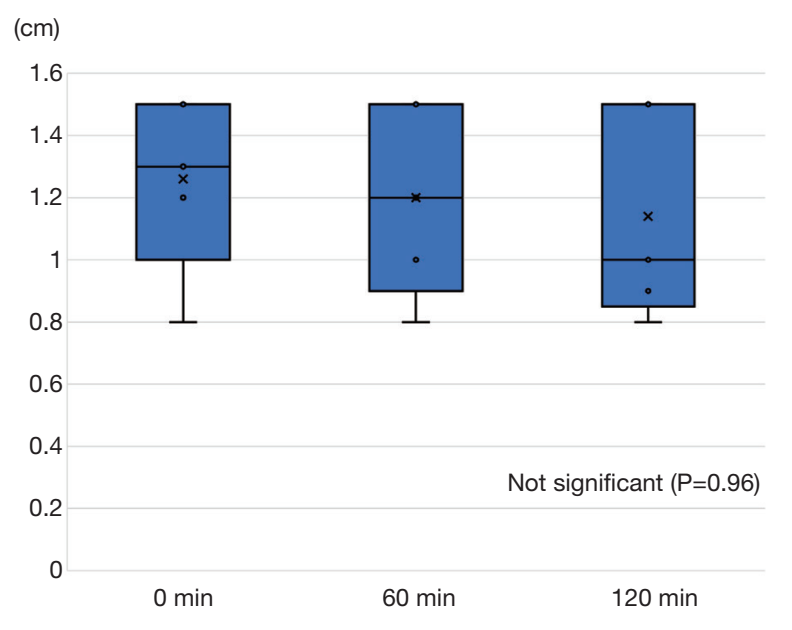

Figure 5 Change over time in the length of the vitamin B2marked area. Measurements were taken every hour, for up to $2 \mathrm{~h}$, starting immediately after thoracotomy.

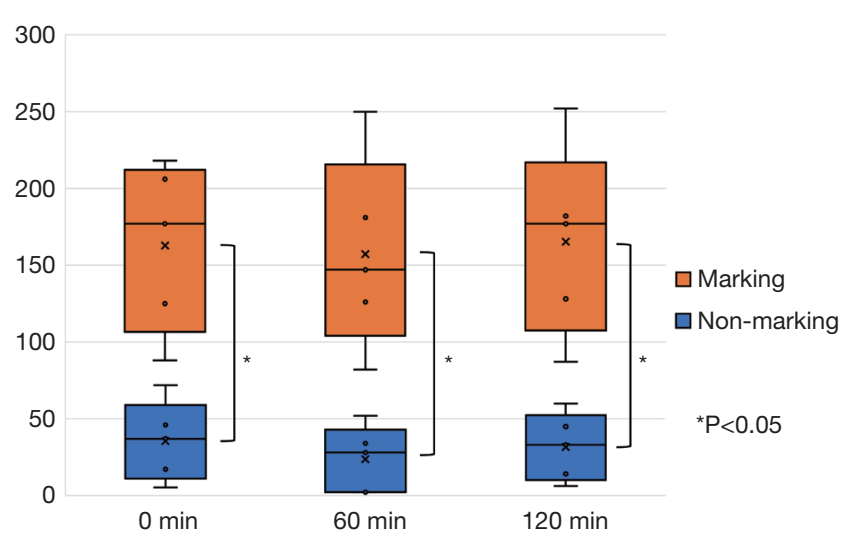

Figure 6 Change over time in fluorescence intensity in the vitamin B2-marked area and the normal lung (non-marked area). Measurements were taken every hour, for up to $2 \mathrm{~h}$, starting immediately after thoracotomy.

which can be lethal.

Transbronchial approaches that use dyes and coils have been reported to avoid lung puncture-related complications. With transbronchial approaches, sites that are difficult to reach using percutaneous approaches can be accessed and multi-site marking can be performed $(12,14,19)$. However, as marking is only possible along the bronchial path, skilled bronchoscope manipulation is necessary to perform accurate marking by selecting a more peripheral branch $(12,14)$. In recent years, Sato et al. reported virtually assisted lung-mapping, which marks multiple sites not only near the tumor but also the lung surface in the surrounding areas using a dye, while providing intraoperative navigation and setting of a cutoff line that secures a sufficient margin (12). Moreover, Nishida et al. reported a marking method that first marks the surface of the body prior to surgery and uses the expansion of the lungs during surgery to mark the lung surface with the dye applied to the chest wall (13). Further, marking with electromagnetic navigation bronchoscopy (20) and a cone-beam CT in hybrid operating rooms (21) have been performed clinically as they are highly accurate marking approaches that do not require lung puncturing. However, if we consider the complexity of the techniques and the need for facilities and human resources, we can infer that widespread use of such technology will take time.

In this study, we used vitamin B2 as a marker and black light as an irradiated light. Vitamin B2 is an autofluorescent substance that exhibits an intense yellow-green color when irradiated with an excitation light of $400 \mathrm{~nm}$. Previously, we reported the identification of lung sentinel lymph nodes and the identification of areas between lung regions (4-6). In the body, vitamin B2 is converted to flavin mononucleotides and FAD and functions as a hydrogen carrier in redox enzymes. Because it is water soluble, vitamin B2 is excreted in urine and is harmless even if ingested excessively; when it is administered at a high dose, the urine color only intensifies (flavinuria). Other dyes, such as indigo carmine and ICG, are used as markers, but there are risks such as allergies and tissue toxicity. ICG cannot be used for patients who have an iodine allergy because it may induce mucosal inflammation and epithelial injury. The benefits of using vitamin B2 are that it is essential for body metabolism, can reduce the risk of allergies, and is cheaper than other dyes $(4,5)$. Furthermore, it can be seen with the naked eye using a black light without using an expensive endoscope system. Black light is used for the detection of bacteria with fluorescent substances in the medical field, as well as for dermatological treatment of conditions like psoriasis, eczema, and white spots. It is safer than UV-B that causes sunburns and UV-C that destroys living tissue, making it safer than sunlight, which carries the whole range of ultraviolet rays, from UV-A to UV-C (22).

In our previous report, the visibility of the vitamin B2-marked area was evaluated using existing endoscopic systems (PDD endoscope system ${ }^{\text {TM }}$, KARL STORZ GmbH and Co., Tuttlingen, Germany) (6), and this is not an excitatory light limited to the maximum excitation wavelength of vitamin B2. The clarity of the marked area may be enhanced by creating an endoscope filter that 
increases the fluorescence of vitamin B2. Black lights also have different peak wavelengths for each product, but the black light used in this study has better visibility than PDD, and we could clearly see the vitamin B2-marked area with the naked eye and thoracoscopy.

Most current marking methods can only identify lung surface marking. We believe that our vitamin B2 marking method in which vitamin B2 is injected under the visceral pleura is a facile and relatively safe method, and we aim to determine its applicability to humans. The vitamin B2-marked area could be confirmed with the naked eye, provided a minimum of $2 \mathrm{~h}$ of visibility, and remained within $1 \mathrm{~cm}$ around the puncture site. Other liquid dye markings have also been found in the injection site for few hours after injection. We could not find any data in the literature regarding diffusion of water-soluble substances after intraparenchymal injection in the lung. However, we assumed that some of the injected vitamin B2 flows into the vasculature and diffuses quickly, but most of the vitamin is retained in the alveoli and stroma, which limit their diffusion. Similar to other percutaneous marking approaches, this method can be implemented without the need of any special device as long as a CT device is available, and because it uses vitamin B2, which is nontoxic to humans and inexpensive, the approach can be easily introduced to any facility. Furthermore, as sufficient visibility can be provided by marking the lungs $2 \mathrm{~h}$ prior to the start of surgery, even if there is no CT device in the operating room, there is enough time to move the patient to an operating room and induce anesthesia after marking, but the process is even easier if a CT device is readily available.

For the hook wire localization method, a puncture of 1 $\mathrm{cm}$ or greater is recommended to prevent the hook wire from dropping and to shorten the puncture time as much as possible, to minimize the risk of air embolism (1). However, our approach is a facile method in which the puncture needle is removed while injecting the vitamin B2 solution after puncturing the visceral pleura, and because filling the syringe and the inner cavity of the puncture needle with FAD sodium can reduce the risk of air embolism, it is a safer method than conventional percutaneous marking. Shimamura et al. reported a lung-marking approach (23) involving a dye and a $25 \mathrm{G}$ needle, which is thinner than the GMS marking needle. Thus, it is highly possible to use vitamin B2 solutions as a marker and reduce the risk of complications associated with lung puncturing even further.

This study had some limitations. First, it was an experiment conducted using healthy pigs, which means that we need to investigate whether similar results can be obtained in human lungs with emphysema. Second, as this study examined the visibility of marking only up to $120 \mathrm{~min}$ after the marking procedure, checking the duration of effective visibility is also necessary. Third, multiple marking is difficult when the patient develops pneumothorax.

In conclusion, we believe that the vitamin B2 lungmarking approach examined in this study will be as effective as the hook wire localization method for lung-marking. In the near future, we plan to conduct clinical trials to evaluate the applicability of this method in humans.

\section{Acknowledgments}

Funding: This work was supported by Japanese Society for the Promotion of Science, Grant/Award Number: JSPS KAKENHI Grant Number JP19K18206.

\section{Footnote}

Conflicts of Interest: The authors have no conflicts of interest to declare.

Ethical Statement: The authors are accountable for all aspects of the work in ensuring that questions related to the accuracy or integrity of any part of the work are appropriately investigated and resolved. The experimental animals were handled in accordance with the "Guidelines for the Care and Use of Laboratory Animals" (approval no. 153644) stipulated by the Kanazawa University, as well as the "Guide for the Care and Use of Laboratory Animals" (Version 8) announced by the National Research Council in 2011.

Open Access Statement: This is an Open Access article distributed in accordance with the Creative Commons Attribution-NonCommercial-NoDerivs 4.0 International License (CC BY-NC-ND 4.0), which permits the noncommercial replication and distribution of the article with the strict proviso that no changes or edits are made and the original work is properly cited (including links to both the formal publication through the relevant DOI and the license). See: https://creativecommons.org/licenses/by-nc-nd/4.0/.

\section{References}

1. Ichinose J, Kohno T, Fujimori S, et al. Efficacy and complications of computed tomography-guided hook wire 
localization. Ann Thorac Surg 2013;96:1203-8.

2. Miyoshi K, Toyooka S, Gobara H, et al. Clinical outcomes of short hook wire and suture marking system in thoracoscopic resection for pulmonary nodules. Eur J Cardiothorac Surg 2009;36:378-82.

3. Sakiyama S, Kondo K, Matsuoka H, et al. Fatal air embolism during computed tomography-guided pulmonary marking with a hook-type marker. J Thorac Cardiovasc Surg 2003;126:1207-9.

4. Matsumoto I, Ohta Y, Waseda R, et al. Vitamin B2 as a tracer for intraoperative pulmonary sentinel node navigation surgery. Anticancer Res 2010;30:4109-14.

5. Waseda R, Oda M, Matsumoto I, et al. A novel fluorescence technique for identification of the pulmonary segments by using the photodynamic diagnosis endoscope system: an experimental study in ex vivo porcine lung. J Thorac Cardiovasc Surg 2013;146:222-7.

6. Ohsima M, Waseda R, Tanaka N, et al. A new fluorescent anatomic pulmonary segmentectomy using PDD endoscope system TM and vitamin B2: evaluation in a clinical setting using living animal. Surg Endosc 2016;30:339-45.

7. Yuvaraj S, Premkumar VG, Vijayasarathy K, et al. Augmented antioxidant status in Tamoxifen treated postmenopausal women with breast cancer on coadministration with Coenzyme Q10, Niacin and Riboflavin. Cancer Chemother Pharmacol 2008;61:933-41.

8. Raiskup F, Spoeri E. Corneal crosslinking with riboflavin and ultraviolet A. I. Principles. Ocul Surf 2013;11:65-74.

9. Nakakuki S. Bronchial tree, lobular division and blood vessels of the pig lung. J Vet Med Sci 1994;56:685-9.

10. Martin-Ucar AE, Nakas A, Pilling JE, et al. A casematched study of anatomical segmentectomy versus lobectomy for stage I lung cancer in high-risk patients. Eur J Cardiothorac Surg 2005;27:675-9.

11. Hsie M, Morbidini-Gaffney S, Kohman LJ, et al. Definitive treatment of poor-risk patients with stage I lung cancer: a single institution experience. J Thorac Oncol 2009;4:69-73.

12. Sato M, Omasa M, Chen F, et al. Use of virtual assisted lung mapping (VAL-MAP), a bronchoscopic multisport dye-marking technique using virtual images, for precise navigation of thoracoscopic sublobar lung resection. J Thorac Cardiovasc Surg 2014;147:1813-9.

13. Nishida T, Fujii Y, Akizuki K. Preoperative marking for peripheral pulmonary nodules in thoracoscopic surgery: a new method without piercing the pulmonary parenchyma. Eur J Cardiothorac Surg 2013;44:1131-3.
14. Nakajima J. Advances in techniques for identifying small pulomonary nodules. Surg Today 2018;24:1742-8.

15. Nagai K, Kuriyama K, Inoue A, et al. Computed tomography-guided preoperative localization of small lung nodules with indocyanine green. Acta Radiol 2018;59:830-5.

16. Watanabe K, Nomori H, Ohtuska T, et al. Usefulness and complications of computed tomography-guided lipiodol marking for fluoroscopy-assisted thoracoscopic resection of small pulmonary nodules: experience with 174 nodules. J Thorac Cardiovasc Surg 2006;132:320-4.

17. Miyoshi T, Kondo K, Takizawa H, et al. Fluoroscopyassisted thoracoscopic resection of pulmonary nodules after computed tomography-guided bronchoscopic metallic coil marking. J Thorac Cardiovasc Surg 2006;131:704-10.

18. Toba H, Kondo K, Miyoshi T, et al. Fluoroscopy-assisted thoracoscopic resection after computed tomographyguided bronchoscopic metallic coil marking for small peripheral pulmonary lesions. Eur J Cardiothorac Surg 2013;44:e126-32.

19. Kojima F, Sato T, Takahata H, et al. A novel surgical marking system for small peripheral lung nodules based on radio flequency identification technology: Feasibility study in a canine model. J Thorac Cardiovasc Surg 2014;147:1384-9.

20. Anayama T, Qiu J, Chan H, et al. Localization of pulmonary nodules using navigation bronchoscope and a near-infrared fluorescence thoracoscope. Ann Thorac Surg 2015;99:224-30.

21. Anayama T, Hirohashi K, Okada H, et al. Simultaneous cone beam computed tomography-guided bronchoscopic marking and video-assisted thoracoscopic wedge resection in a hybrid operating room. Thorac Cancer 2019;10:579-82.

22. Sliney DH, GilbertII DW, Lyon T. Ultraviolet safety assessments of insect light traps. J Occup Environ Hyg 2016;13:413-24.

23. Shimamura Y, Sasaki S, Shimohira M, et al. New technique of percutaneous CT fluoroscopy-guided marking before video-assisted thoracoscopic surgery for small lung lesions: feasibility of using a 25-gauge needle without local anaesthesia. Br J Radiol 2018;91:20170692.

Cite this article as: Tanaka Y, Matsumoto I, Takata M, Saito D, Yoshida S, Tamura M, Koda W, Waseda R, Takemura H. Vitamin B2 lung-marking method using black light irradiation. J Thorac Dis 2020;12(3):651-658. doi: 10.21037/jtd.2020.01.06 